\title{
Implementation and Analysis of Scheduling Algorithms for LTE Network
}

\author{
Radhika R. Kulkarni \\ Department of Computer Science and Engineering \\ KLS Gogte Institute of Technology \\ Belagavi, Karnataka, India
}

\author{
P. M. Pujar \\ Department of Computer Science and Engineering \\ KLS Gogte Institute of Technology \\ Belagavi, Karnataka, India
}

\begin{abstract}
Long Term Evaluation (LTE) network, which primarily focuses on providing the high-speed communication for mobiles and other terminals/devices. In LTE the main mechanism is to process the traffic, which is done by the Packet Scheduler. Packet Scheduler is mainly responsible to send the packets to user nodes depending on the algorithm that is implemented at the Base Station. Packet scheduler is also responsible for queue management, scheduling of the packets, sending the packets to user nodes by allocating resources to them. Therefore, the key idea of paper is to implement a Scheduling Algorithm which meets the QoS requirements of LTE network. The performance analysis is done by comparing the NewQueue scheduling algorithm with the DropTail algorithm. Metrics that are used for comparing the performance are Throughput, Delay and Jitter.
\end{abstract}

\section{Keywords}

LTE, Scheduling Algorithm, QoS, Packet Scheduler, NewQueue, DropTail

\section{INTRODUCTION}

As there is a increasing need for on demand network service such as Voice Calling, Video Streaming, Video Calling and Web browsing with constraints on delay and bandwidth specifications, which imposes the new challenges for designing the upcoming generation of cellular network[2]. Hence, the Long Term Evaluation(LTE) networks were introduced so that they can meet the new requirements/challenges posed on the cellular network . Which mainly aims at achieving greater performance and defining a new packet optimized architecture for the radio access network.

In order to complete the needs of the current on demand and variety of applications, LTE networks have been designed by taking into the consideration the requirements for on demand Voice and Video application so that it can overtake the $3 \mathrm{G}$ networks. LTE tries to give the better data rates compare to the other technologies. The Peak Data Rates that are allowed for both the up-link and down-link in LTE are 100 and 50 Mbps respectively [7] .

\subsection{Packet Scheduler In LTE}

Key idea behind implementing scheduling algorithm is to achieve the performance gain in the network. In any network the scheduling is performed by the Scheduler, which is present at base station and performs many tasks such as managing the queue then scheduling the data packets and transmitting those packets to the user nodes and allocating resources to the users.

These all tasks are performed by the scheduler by making use of the scheduling algorithm which is available at the base station.
A scheduler in the LTE network has some of the important objectives[9]:

- Link Adaption

- Rate Control

- Packet Scheduler and Resource Assignment

The scheduling types that are supported by the LTE network are[9]:

Dynamic Scheduling : On every time interval in the dynamic scheduling, the MAC checks for the user nodes that can be scheduled. This decision is taken by considering the two things. First, Whether user node has any data to be scheduled in the buffer. Second, Whether there are enough number of resource blocks available to schedule the particular user.

Persistent Scheduling : Here, the packets/data are scheduled in the fixed manner which is similar to the circuit switching. Same amount of resource blocks are assigned to all scheduled users.

Semi-Persistent Scheduling : This is the combination of both dynamic and persistent scheduling. Hence it is called as hybrid scheduling. It tries to give better performance compare to both dynamic and persistent scheduling types.

\section{LITERATURE SURVEY}

LTE network aims at providing the fast services to the mobiles in the high mobility situations. To accomplish this goal LTE includes some Radio Resource Management techniques. In the paper [10], authors have performed an analysis on the existing scheduling algorithms available for LTE such as Proportional Fair algorithm, Exponential-Rule algorithm, Exponential-PF algorithm, Maximum Largest Weighted Delay First algorithm, Logarithmic-Rule algorithm and Frame-Level Scheduler for LTE down link direction. Execution is performed for the single cell with some barrier condition and trying to expand number of clients and their speed.

In the recent times, wireless networks need to be associated with the remote client devices along with its existing system. QoS is the essential parameter that will be dealt with excellently when the exchange of information is taking place between the nodes. In WiMax, QoS is determined correctly at the MAC layer though the data transfer capacity related calculations related to the QoS is not clearly mentioned in the IEEE-802.16[11].

In the paper [11], authors have thought about the different available calculations and try to clarify the unique issues related to these calculations. Apart from these authors proposed a new calculation for data transfer distribution for IEEE 802.16 WiMax mainly to enhance the QoS.

Long Term Evolution Advanced(LTE-A) is the fastest growing technology. LTE - Advanced is the new form of the 
LTE network. LTE-A tries to improve the performance by providing the new and enhanced administrative ability, improved system execution by introducing the new methods. In LTE network the information is transmitted in term of packets to the mobile nodes. LTE encourages traffic like video streaming, voice calling etc. Proper planning in terms of calculations related to transmitting theses packets is the key part of LTE systems[12].

In the paper [12], authors mainly deal with planning to perform some of the calculation related to the LTE down link scheduling. Some important task that has to be performed by LTE-A are: to upgrade the LTE - Advanced for the heterogeneous networks, to perform the data transmission with respect to multipoint, utilization of the resources in the remote system. LTE- Advanced is used to improve the radio recourse allocation in the system.

LTE network was proposed by $3 \mathrm{GPP}$ as the $3.9 \mathrm{G}$ technology. LTE tries to provide a faster response to the mobile nodes which are having the high data transmission request. To improve the performance related to the video and voice data request, LTE include a number of RRM systems. To achieve this goal the base station need to have a proper scheduling algorithm and a scheduler which is mainly held responsible for resource scheduling and resource allocation to the mobile nodes. In such a situation radio resource allocation plays a very important role which is performed by RRM. In the paper [13], authors explain the way in which the resource allocation is done in the LTE down link direction and also the brief explanation about LTE scheduling algorithm is specified in the paper.

In LTE network, scheduling and recourse allocation are the important tasks. So that the data transmission can be constant which will help in improving the performance of the LTE network. But achieving the constant data transmission for the voice and video services is bit difficult because they will be dealing with a live streaming data. To overcome these difficulties, authors in [14] have introduced a new scheduler named as WE-MQS which stands for Wideband E-model Channel and QoS Aware, designed for dealing with voice services in LTE downlink direction. The voice data may be very sensitive to the network problem such as delay, noise etc. The proposed scheduler tries to extend the maximum queue size. And it also considers maintaining the Mean Opinion Score value so that the scheduler can recognize the clients which have higher data rate need.

In the paper [15], authors mainly focus on understanding the requirements for LTE network as per the International Mobile Telecommunication-Advanced. LTE should have $1 \mathrm{Gbps}$ and $500 \mathrm{Mbps}$ for down link and up link respectively. LTE must also support OFDMA, Carrier Aggregation, Multi Input and Multi Output, Techniques for Coordinated Transmission in Multi-Point.

\section{OBJECTIVE}

LTE has a growing demand in the current cellular network because it provides high data rate to the user nodes compared to the other technologies such as $3 \mathrm{G}$ technologies. To provide the high performance to users LTE should have a better scheduling strategies. Scheduling is mainly handled by the scheduler that is present at the base station. Scheduling algorithm is the key mechanism which helps in improving the LTE network performance. Therefore, the paper mainly aims at implementing the new scheduling algorithm NewQueue, which is modification of the previous scheduling algorithm available for LTE network. The NewQueue algorithm mainly focuses on improving the performance of the LTE network by increasing the throughput and reducing the delay and jitter while transferring the packets form base stations to users.

\section{SYSTEM MODEL}

General packet scheduling method for LTE down link network is shown in Fig 1 [10]. In LTE network, the task of packet scheduling is performed in the base station that is the eNodeB (evolved NodeB). There will be a separate buffer maintained for each and every user at the base station (eNodeB). Which are used to store the packets coming from server. The main component present in the eNodeB and performs the task of scheduling is the scheduler. This scheduler will be scheduling these packets depending on the scheduling algorithm/ scheduling criteria that is also present at the base station.

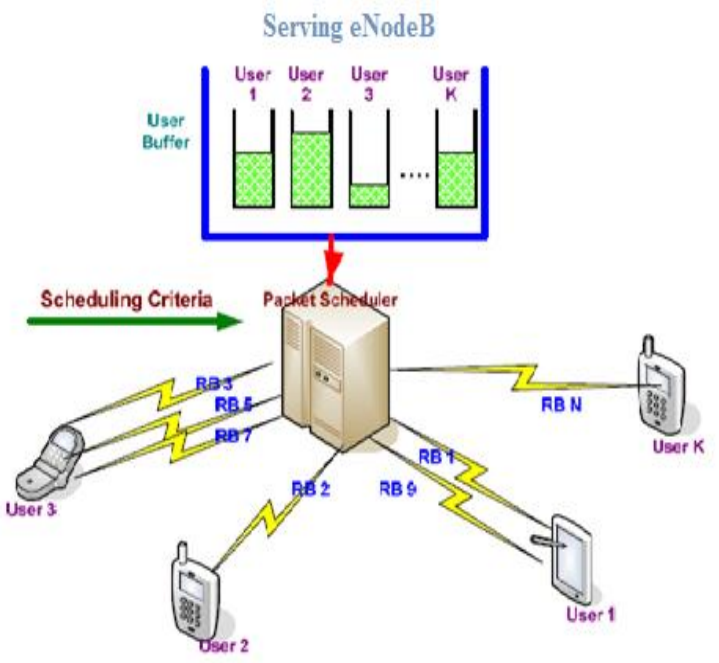

Fig 1: Packet Scheduling Model

Scheduler is responsible not only for packet scheduling but also for queue management. At every time interval that is at every second scheduler schedules the packets present at the buffer depending on the scheduling criteria. The packets need to be scheduled in a faster manner so that buffer can have enough space to store the next set of incoming packets. Because if the user buffers are full then the incoming packets will be dropped due to which users may face the data loss problem.

\subsection{DropTail Algorithm}

The conventional method that is used to manage the queue is to set the maximum queue size that is in terms of packets for each queue separately. Queue can accept and store the packets until it reaches the maximum size. Once the queue exceeds the maximum limit then the successive incoming packets will be dropped until some packets are transmitted and there is sufficient space in queue to store the packets coming from server.

This well known method of managing queue is known as Drop Tail, because the packets that have arrived recently will be dropped first when queue is filled completely( that is the packet at the tail of the queue will be dropped). In this technique each packet has the same priority there is no differentiation done in the traffic[28]. This method was used in the Internet for many years. But this technique has some of the draw backs[28]: lock-out and steady queue size.

In short the DropTail technique does not have a effective queue management. As the network demand increases the 
quantity of data that has to be transferred through the links increases, which cant not be left unmanaged. Hence a NewQueue algorithm has been proposed to handle the queue efficiently and to reduce the packet loss in the network compared to the DropTail technique.

\subsection{NewQueue Algorithm}

NewQueue algorithm is mainly used to enhance the performance of the complete network. In [1] authors have focused on calculating the scheduling metric for each and every packet that is $\mathrm{W}_{\mathrm{i}, \mathrm{j}}$, which is mainly used for scheduling the packets. This algorithm also prioritize the packets depending on the traffic type so that higher priority packets can be scheduled first and does not face any packet loss.

Considering the characteristics of traffic, mainly with respect to the delay need, a scheduling process have to consider a variety of factors that is the packets need to be scheduled within the specified time limit, to prevent them from being discarded as it may reach the deadline. So that the packets are scheduled soon and there is enough space in the buffer to store the next set of packets, and queue does not drop the packets due to in sufficient space[1].

\section{IMPLEMENTATION DETAILS}

Paper mainly includes 2 modules :

\subsection{DropTail Algorithm}

This module consist of files such as newQueue.tcl, which mainly defines the LTE cellular network. Throughput.awk , Jitter.awk, Delay.awk, these are the scripting files written to process the trace file which mainly has all the data related to each and every transaction. Test file, which outputs the metric values on the terminal.

\subsection{NewQueue Algorithm}

It has the similar files as of NewQueue algorithm, such as DropTail.tcl, AWK files for Jitter, Throughput and Delay and a Test file. But the major difference is that these files are written to show the DropTail queue scheduling mechanism. Where in the files in NewQueue mainly focuses on showing the proposed strategy.

To analyze the performance DropTail and NewQueue algorithm, NS-2 Simulation tool is used. Topology that is used for both the algorithm is identical to the network architecture of LTE network. Various components in the topology are illustrated as below, Node 0 is the evolved NodeB (eNB) or base station, Node 1 the Serving Gateway (S-GW) or Mobility Management Entity (MME), and Node 2 is server. Rest all the nodes are user equipments (UE). The simulation ends time is set to $30 \mathrm{~m}$.

\section{RESULTS AND DISCUSSION}

Performance metrics are the one, which are mainly used to calculate the performance of entire network depending on some mathematical equations. Performance metrics which are used in the paper for analyzing the performance of NewQueue and DropTail algorithm are Throughput, Delay and Jitter.

\subsection{Throughput}

In scheduling algorithm, Throughput refers to the efficiency of the algorithm. Efficiency is the total amount of packets that are successfully transmitted to the user nodes from the base station without any packet loss.

Fig 2 shows the comparison between NewQueue and DropTail algorithm by considering Throughput as the performance metric. From the above graph it can be noticed that NewQueue algorithm performs better and efficiently compared to the DropTail algorithm.

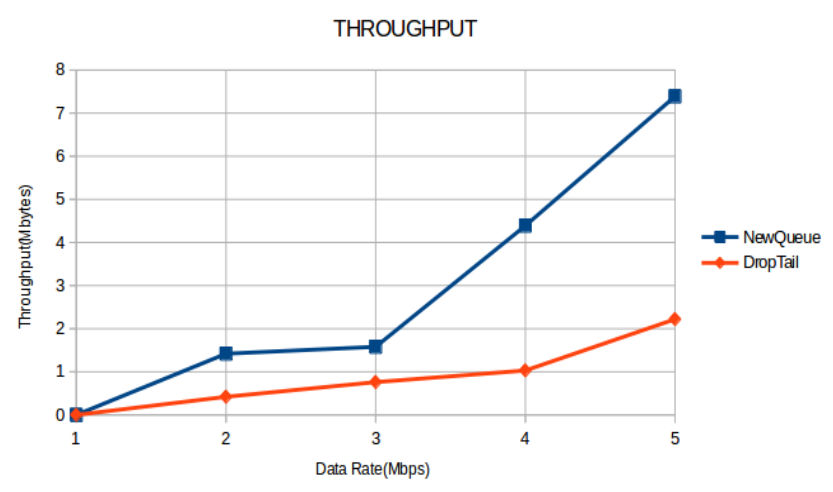

Fig 2: Comparison Graph for Throughput

In the above graph $\mathrm{X}$ axis represent the Data Rate in terms of Mbps and $\mathrm{Y}$ axis represents Throughput in M Bytes. The proposed NewQueue algorithm achieves greater throughput results than the existing DropTail algorithm as data rate(Mbps) increases.

\subsection{Delay}

Delay in the scheduling algorithm is, the total time taken for transmitting the packets from Base Station to User Nodes. Each packet that is sent to the user node will be associated with the delay value.

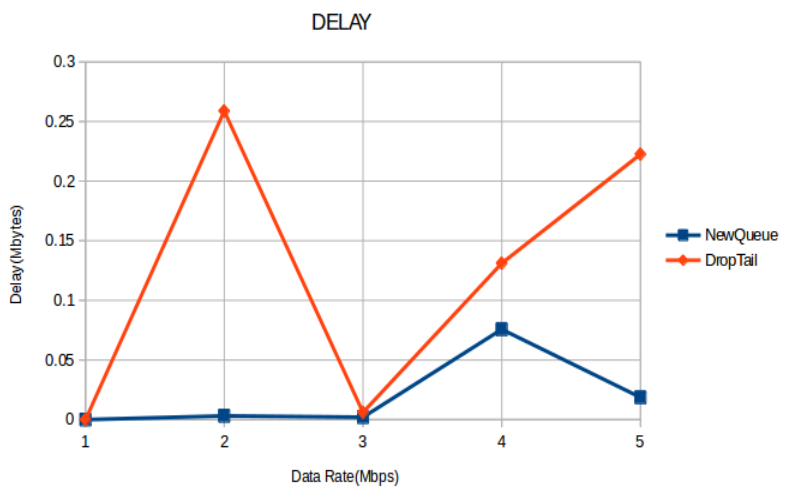

Fig 3: Comparison Graph for Delay

Fig 3 shows comparison of NewQueue and DropTail algorithm by taking into consideration Delay as the metric. By considering the graph results it can be said that NewQueue Algorithm takes less time to transmit all the packets compared to the DropTail algorithm.

In graph $\mathrm{X}$ axis represents the Data Rate (Mbps) and $\mathrm{Y}$ axis represents Delay (M Bytes). The NewQueue algorithm transmits all packets from base station to users in the lesser amount of time than the DropTail algorithm.

\subsection{Jitter}

Jitter can be defined as the variations in the time of receiving the packets. The base station will be continuously sending the packets to the user nodes. But user nodes may face a difficulties in receiving those packets in the continues manner due to many reasons such as congestion of network, improperly queuing the packets or due to errors in configuration. 


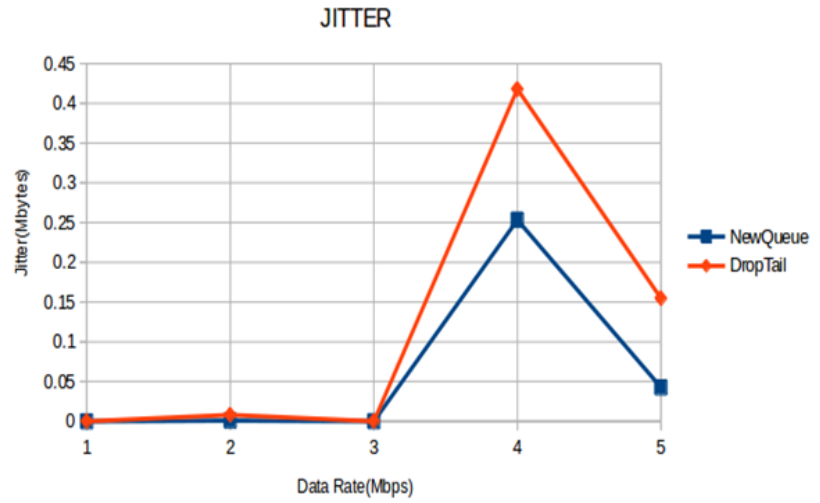

Fig 4: Comparison Graph for Jitter

Fig 4 represents the comparison between NewQueue algorithm and DropTail algorithm by considering Jitter as metric. From the graph it can be analyzed that in the proposed algorithm, the user nodes face lesser delay in receiving the packets than the existing algorithm.

In graph $\mathrm{X}$ axis represent the Data Rate (Mbps) and $\mathrm{Y}$ axis represent Delay (M Bytes). The NewQueue algorithm helps the user in receiving the packets more efficiently by reducing the delay.

\section{CONCLUSION}

In this paper a NewQueue scheduling algorithm is implemented along with a DropTail scheduling algorithm. The performance analysis is carried out by comparing the NewQueue algorithm with the DropTail algorithm by considering Throughput, Delay and Jitter as the performance metrics. Based on simulation results and graphs it can be analyzed that the NewQueue algorithm is more efficient compared to the existing DropTail algorithm with respect to Delay, Jitter and Throughput. Hence NewQueue scheduling algorithm can be used for improving the performance of entire LTE network.

\section{FUTURE WORK}

As a part of the future work NewQueue scheduling algorithm may be used to schedule the packets related to real-time data such as voice calling and video streaming services. The NewQueue Algorithm can be analyzed by using the other metrics such as PLR and Spectral Efficiency. And the comparison of NewQueue algorithm can be done with other algorithms such as PF, M-LWDF, VT-M-LWDF and QueueHOL-MLWDF for LTE network.

\section{REFERENCES}

[1] Dardouri Samia, Bouallegue Ridha, "A New Scheduling Algorithm for Real- Time Communication in LTE network", 29th International Conference on Advanced Information Networking and Applications Workshops, 2015.

[2] F. Capozzi, G. Piro, L. A. Grieco, G. Boggia and P. Camarda, Downlink "Packet Scheduling in LTE Cellular Networks: Key Design Issues and a Survey", IEEE Communications Surveys and Tutorials, Volume: 15, Issue: 2, 2013.

[3] 3GPP, Tech. Specif. Group Radio Access Network Requirements for Evolved UTRA (E-UTRA) and Evolved UTRAN (E-UTRAN), 3GPP TS 25.913.
[4] Iturralde Ruiz, Performances des Rseaux LTE, thesis Institut National Polytechnique de Toulouse (INP Toulouse) 2012.

[5] Technical White paper: LTE eNodeB MAC Scheduler Introduction, by Roke Manor Research.

[6] M. Andrews, K. Kumaran, K. Ramanan, et al. "Providing quality of service over a shared wireless link", IEEE Commu. Mag., vol. 39, no. 2, pp. 150 154, Feb. 2001.

[7] White Paper, Long Term Evolution (LTE): A Technical Overview, 2008.

[8] 3GPP, Tech. Specif. Group Radio Access Network Physical Channel and Modulation (Release 8), 3GPP TS 36.211 .

[9] Lakshmikishor Nittala, Preeti Kanwar Singh Rekhi, Sukhvinder Singh Malik and Rahul Sharma, "LTE Scheduler - A Definitive Approach", International Journal of Inventive Engineering and Sciences (IJIES), ISSSN: 2319-9598, Volume-2, Issues-4, March 2014.

[10] Farhana Afroz, Roshanak Heidery, Maruf Shehab, Kumbesan Sandrasegaran and Sharmin Sultana Shompa, "Comparative Analysis Of Downlink Packet Scheduling Algorithms In 3GPP LTE Networks", International Journal of Wireless and Mobile Networks (IJWMN) Vol. 7, No. 5, October 2015.

[11] Avinash Kaur, "Bandwidth Allocation Scheduling Algorithms for IEEE 802.16 WiMax Protocol to Improve QoS: A Survey", International Journal of Computer Applications (0975 - 8887) Volume 98- No.11, July 2014.

[12] Ammar Hafeez, Mohammad Masoom Zafar, Sir Ahmad Mudassir, "A Survey on Analysis and Design of Scheduling Algorithm for LTE-Advanced Heterogeneous Networks", COMSATS Institute of Information Technology, LHR Pakistan.

[13] Davinder Singh, Preeti Singh, "Radio Resource Scheduling in 3GPP LTE: A Review", International Journal of Engineering Trends and Technology (IJETT) - Volume 4 Issue 6- June 2013.

[14] Hang Nguyen, "WE-MQS: A new LTE Downlink Scheduling Scheme for Voice Services based on User Perception", International Journal of Computer Applications (0975 - 8887) Volume 142 - No.10, May 2016.

[15] S. Radhakrishnan, S. Neduncheliyan and K. K. Thyagharajan, "A Review of Downlink Packet Scheduling Algorithms for Real Time Traffic in LTEAdvanced Networks", Indian Journal of Science and Technology, Vol 9, January 2016.

[16] Chadchan. S. M. and Akki. C. B., "A Fair Downlink Scheduling Algorithm for 3GPP LTE Networks", I. J. Computer Network and Information Security, pp. 34 41, May 2013.

[17] Xiaowei LI, Bingbing LI, Bing LAN, Min HUANG, and Guanghui YU, "Adaptive PF Scheduling Algorithm in LTE Cellular System", IEEE International Conference on Information and Communication Technology Convergence (ICTC), Nov. 2010, pp. 501 - 504. 
[18] Tian Xie and Xiaowei Hui, "LTE System Scheduling Algorithm based on Utility Function", IEEE, Nov. 2011, pp. 370 - 373.

[19] Mohammad T. Kawser, Hasib M. A. B. Farid, Abduhu R. Hasin, Adil M. J. Sadik, and Ibrahim K. Razu, "Performance Comparison between Round Robin and Proportional Fair Scheduling Methods for LTE", International Journal of Information and Electronics Engineering, Vol. 2, No. 5, pp. 678 - 681, September 2012.

[20] Donald Parruca, Marius Grysla, Simon Gortzen, and James Gross,, "Analytical Model of Proportional Fair Scheduling in Interference-limited OFDMA/LTE Networks", Vehicular Technology Conference (VTC Fall), 2013.

[21] Tolga Girici, Chenxi Zhu, Jonathan R. Agre, and Anthony Ephremides, "Proportional Fair Scheduling Algorithm in OFDMA-Based Wireless Systems with QoS Constraints", International Journal of Information and Electronics Engineering, Journal of Communications and Networks, Vol. 12, No. 1, pp. 30 - 42, Feb. 2010.

[22] Zhishui Sun, Changchuan Yin, and Guangxin Yue, "Reduced-Complexity Proportional Fair Scheduling for OFDMA Systems", IEEE International Conference on Communications, Circuits and Systems Proceedings, Vol. 2, pp. 1221 - 1225, June 2006.

[23] Oana IOSIF, and Ion BANICA, "Performance Analysis OF Downlink LTE USING System Level Simulator", U.P.B. Sci. Bull., Series C, Vol. 75, Jan. 2013.

[24] Stojan Kitanov, Toni Janevski , "Performance Evaluation of Scheduling Strategies for LTE Networks in Downlink Direction", in Proceedings of the XI International Conference ETAI 2013, 26th -28th of September 2013.
[25] J. H. Rhee, J. M. Holtzman, D. K. Kim, "Scheduling of real/non-real time services: adaptive EXP/PF algorithm", VTC 2003-Spring. The 57th IEEE Semiannual, vol. 1, pp.462 466, 2003.

[26] M. Iturralde, A. Wei, T. Yahiya, and A. Beylot, "Performance study of multimedia services using virtual token mechanism for resource allocation in LTE networks", in IEEE Vehicular Technology Conference(VTC), Sept. 2011, pp. 15.

[27] P. Ameigeiras, J. Wigard, and P. Mogensen, "Performance of the m-lwdf scheduling algorithm for streaming services in hsdpa", IEEE Trans. Veh. Technol. Conf., vol. 2, pp. 999-1003, Sep. 2004. Los Angeles, USA.

[28] Serhat Özekes, "Evaluation Of Active Queue Management Algorithms", stanbul Ticaret Üniversitesi Fen Bilimleri Dergisi Y1l:4 Say1:7 Bahar 2005/1 s.123140 .

[29] Mrs. Saba Siraj, Mr. Ajay Kumar Gupta, Mrs. Rinku Badgujar, "Network Simulation Tools Survey", International Journal of Advanced Research in Computer and Communication Engineering Vol. 1, Issue 4, June 2012.

[30] Pallavi S. Katkar, Dr. Vijay R. Ghorpade, "Comparative Study of Network Simulator: NS2 and NS3", International Journal of Advanced Research in Computer Science and Software Engineering, Volume 6, Issue 3, March 2016.

[31] NS2 installation steps - http://ns2-ubuntu.blogspot.in

[32] NS2 Installation Steps- http://surajpatilworld.blogspot.in/ 2015/02/step-by-step installation-of-ns-234-on.html

[33] NS2 with LTE patch - www.linuxquestions.org 